\title{
An accurate approach based on the orthonormal shifted discrete Legendre polynomials for variable-order fractional Sobolev equation
}

\author{
M.H. Heydari ${ }^{1 *}$ (I) and A. Atangana ${ }^{2,3}$
}

"Correspondence:

heydari@sutech.ac.ir

${ }^{1}$ Department of Mathematics,

Shiraz University of Technology,

Shiraz, Iran

Full list of author information is

available at the end of the article

\section{Springer}

\begin{abstract}
This paper applies the Heydari-Hosseininia nonsingular fractional derivative for defining a variable-order fractional version of the Sobolev equation. The orthonormal shifted discrete Legendre polynomials, as an appropriate family of basis functions, are employed to generate an operational matrix method for this equation. A new fractional operational matrix related to these polynomials is extracted and employed to construct the presented method. Using this approach, an algebraic system of equations is obtained instead of the original variable-order equation. The numerical solution of this system can be found easily. Some numerical examples are provided for verifying the accuracy of the generated approach.
\end{abstract}

Keywords: Variable-order time fractional Sobolev equation; Orthonormal shifted discrete Legendre polynomials; Nonsingular variable-order fractional derivative

\section{Introduction}

Over the past decades, the subject of fractional calculus (as a generalization of the classical calculus) has been widely studied [1-3]. In fact, fractional derivative and integral operators, due to higher degree of freedom in comparison to the classical operators as well as their memory and nonlocal properties, have received many applications in various problems [4]. For instance, some important works related to recent developments in fractional calculus and its applications can be found in [5-10]. The reader should note that the most important issue about problems involving such operators is finding their exact solutions, which is often very difficult and may even be impossible. This fact has led to the use of numerical methods as a convenient alternative to solve this drawback. Some numerical methods that have recently been applied to solve such problems can be found in [11-18].

Given that the order of fractional operators is permissible to take any value, a more general generalization is that the order of fractional operators be a definite function of the variables in the problem [19]. In fact, fractional operators of variable order (VO) can be utilized for more accurate modeling of real-world phenomena [20, 21]. The remarkable point about such operators is that their memory property is more evident [22]. Some

(c) The Author(s) 2021. This article is licensed under a Creative Commons Attribution 4.0 International License, which permits use, sharing, adaptation, distribution and reproduction in any medium or format, as long as you give appropriate credit to the original author(s) and the source, provide a link to the Creative Commons licence, and indicate if changes were made. The images or other third party material in this article are included in the article's Creative Commons licence, unless indicated otherwise in a credit line to the material. If material is not included in the article's Creative Commons licence and your intended use is not permitted by statutory regulation or exceeds the permitted use, you will need to obtain permission directly from the copyright holder. To view a copy of this licence, visit http://creativecommons.org/licenses/by/4.0/. 
problems that have recently been modeled by such operators can be found in [23, 24]. However, similar to constant-order fractional equations, the major challenge in dealing with $\mathrm{VO}$ fractional equations is finding their analytical solutions, which is often impossible. For this reason, in recent years, many numerical approaches have been constructed to solve this category of problems. For instances, see [25-29].

The Sobolev equation is a well-studied partial differential equation which has been frequently utilized in the fluid dynamics to express the fluid motion through rock or soil, and other media [30]. This equation is a special form of the Benjamin-Bona-MahonyBurgers problem, where the coefficients of nonlinear term and both first-order derivatives are zero [31]. Many applications of the Sobolev equation have been reported in moisture migration in soil [32], thermodynamics [33], and fluid motion [33]. There are many approaches that have been applied to solve various types of the Sobolev equation in recent years. For instances, see [30, 31, 34-37].

Recently, the author of [38] introduced a new nonsingular VO fractional derivative, where the Mittag-Leffler function is its kernel. As far as we know, there is no previous VO fractional version of the Sobolev problem. This motivates us to pursue the following goals:

- Defining a VO fractional prescription of the Sobolev equation using the nonsingular fractional derivative expressed in [38].

- Constructing a highly accurate method based upon the orthonormal shifted discrete Legendre polynomials (DLPs) for this equation.

So, we concentrate on the problem

$$
\begin{gathered}
{ }_{0}^{H H} \partial_{\tau}^{\zeta(\tau)} \theta(y, \tau)-\mu_{0}^{H H} \partial_{\tau}^{\zeta(\tau)} \theta_{y y}(y, \tau)-v \theta_{y y}(y, \tau)=\varphi(y, \tau), \\
\zeta(\tau) \in(0,1),(y, \tau) \in\left[0, y_{b}\right] \times\left[0, \tau_{b}\right],
\end{gathered}
$$

under the initial and boundary conditions

$$
\theta(y, 0)=\hat{\theta}(y), \quad \theta(0, \tau)=\tilde{\theta}_{0}(\tau), \quad \theta\left(y_{b}, \tau\right)=\tilde{\theta}_{1}(\tau)
$$

where $\theta(\cdot, \cdot)$ is the undetermined solution, $\mu$ and $v$ are positive constants, $\zeta(\cdot)$ is a continuous function in its domain, and $\varphi(\cdot, \cdot), \hat{\theta}(\cdot), \tilde{\theta}_{0}(\cdot)$, and $\tilde{\theta}_{1}(\cdot)$ are given functions. Also, ${ }_{0}^{H H} \partial_{\tau}^{\zeta(\tau)} \theta(y, \tau)$ is the VO fractional derivative of order $\zeta(\tau)$ with respect to $\tau$ in the HeydariHosseininia $(\mathrm{HH})$ sense of the functions $\theta(y, \tau)$ [38]. This equation can have useful applications in many applied problems, such as the transport phenomena of humidity in soil, the heat conduction phenomena in different media, and the porous theories concerned with percolation into rocks with cracks. Note that in the case of $\zeta(\tau)=1$, this problem reduces to the classical Sobolev problem.

One good idea for solving fractional functional equations is employing polynomials as basis functions to construct numerical methods. This is important for two reasons: First, the computation of the fractional derivative and integral of these functions is easy; and second, if the solution of the problem under study is sufficiently smooth, high-precision solutions can be achieved. Basis orthogonal polynomials are classified into discrete and continuous kinds regarding the method of calculating their expansion coefficients [39]. Unlike continuous polynomials, the expansion coefficients of which are calculated by integrating (in most cases numerically), the expansion coefficients of discrete polynomials 
are calculated accurately using a finite summation. In recent years, discrete polynomials have been extensively applied for solving diverse problems. For instances, see [39-47].

This study applies the orthonormal shifted DLPs for solving the Sobolev equation (1.1) subject to conditions (1.2). To this end, a new fractional matrix related to the $\mathrm{VO}$ fractional differentiation of these polynomials is obtained and applied for generating a numerical technique for this problem. The intended approach is constructed using these polynomials expansion and the tau technique. This technique converts the VO fractional problem into an algebraic system of equations that readily can be handled. Note that since it is easier to obtain the operation matrix of $\mathrm{VO}$ fractional derivative of the orthonormal shifted DLPs than continuous polynomials, we have considered these discrete polynomials as basis functions for solving this $\mathrm{VO}$ fractional problem.

Organization of this article is as follows: The $\mathrm{VO}$ fractional derivative in the $\mathrm{HH}$ sense is reviewed in Sect. 2. The orthonormal shifted DLPs are reviewed in Sect. 3. Some matrix equalities are obtained in Sect. 4. The computational approach is explicated in Sect. 5. Numerical examples are given in Sect. 6. Conclusion of this study is provided in Sect. 7.

\section{Preliminaries}

Here, we review the definition of the VO fractional differentiation used in this study. First of all, we express the definition of the Mittag-Leffler function that is given in [4] by

$$
\mathbf{E}_{a, b}(\tau)=\sum_{j=0}^{\infty} \frac{\tau^{j}}{\Gamma(j a+b)}, \quad a, b \in \mathbb{R}^{+}, \tau \in \mathbb{C} .
$$

Please remember that for $b=1$ it is considered as $\mathbf{E}_{a}(\tau)=\mathbf{E}_{a, 1}(\tau)$. The VO fractional derivative of order $\zeta(\tau) \in(0,1)$ (where $\zeta(\tau)$ is a continuous function on its domain) in the $\mathrm{HH}$ sense of the function $\theta(\tau)$ is given in [38] as follows:

$$
{ }_{0}^{H H} D_{\tau}^{\zeta(\tau)} \theta(\tau)=\frac{1}{1-\zeta(\tau)} \int_{0}^{\tau} \mathbf{E}_{1}\left(\frac{-\zeta(\tau)(\tau-s)}{1-\zeta(\tau)}\right) \theta^{\prime}(s) d s, \quad \tau>0 .
$$

The above definition results in

$$
{ }_{0}^{H H} D_{\tau}^{\zeta(\tau)} \tau^{r}= \begin{cases}0, & r=0 \\ \frac{r !}{1-\zeta(\tau)} t^{r} \mathbf{E}_{1, r+1}\left(-\frac{\tau \zeta(\tau)}{1-\zeta(\tau)}\right), & r \geq 1\end{cases}
$$

where $r \in \mathbb{Z}^{+} \bigcup\{0\}$.

\section{Orthonormal shifted discrete Legendre polynomials}

The orthonormal shifted DLPs are defined over $\left[0, \tau_{b}\right]$ as follows [44]:

$$
L_{\tau_{b}, i}(\tau ; N)=\frac{1}{\sqrt{\sigma(i, N)}} \sum_{k=0}^{i} \sum_{m=0}^{k}(-1)^{k}\left(\begin{array}{c}
i \\
k
\end{array}\right)\left(\begin{array}{c}
i+k \\
k
\end{array}\right)\left(\frac{N}{\tau_{b}}\right)^{m} \frac{S_{k}^{(m)}}{N^{(k)}} \tau^{m}, \quad i=0,1, \ldots, N,
$$

where

$$
\sigma(i, N)=\frac{(N+i+1)^{(i+1)}}{(2 i+1) N^{(i)}}
$$


$S_{k}^{(m)}$ s are the first type Stirling numbers,

$$
N^{(k)}= \begin{cases}1, & k=0, \\ N(N-1)(N-2) \ldots(N-k+1), & k \geq 1,\end{cases}
$$

and $\left(\begin{array}{l}i \\ k\end{array}\right)$ is the binomial coefficient. These polynomials can be utilized for approximating any continuous function $\theta(\tau)$ over $\left[0, \tau_{b}\right]$ as follows:

$$
\theta(\tau) \simeq \sum_{i=0}^{N} e_{i} L_{\tau_{b}, i}(\tau ; N) \triangleq \mathbf{E}^{T} \Psi_{\tau_{b}, N}(\tau)
$$

where

$$
\mathbf{E}=\left[\begin{array}{llll}
e_{0} & e_{1} & \ldots & e_{N}
\end{array}\right]^{T},
$$

in which

$$
e_{i}=\sum_{r=0}^{N} \theta\left(\frac{\tau_{b}}{N} r\right) L_{\tau_{b}, i}\left(\frac{\tau_{b}}{N} r ; N\right)
$$

and

$$
\Psi_{\tau_{b}, N}(\tau)=\left[L_{\tau_{b}, 0}(\tau ; N) L_{\tau_{b}, 1}(\tau ; N) \ldots L_{\tau_{b}, N}(\tau ; N)\right]^{T} .
$$

Likely, a continuous function $\theta(y, \tau)$ defined over $\left[0, y_{b}\right] \times\left[0, \tau_{b}\right]$ can be approximated by the orthonormal shifted DLPs as

$$
\theta(y, \tau) \simeq \sum_{i=0}^{M} \sum_{j=0}^{N} \theta_{i j} L_{y_{b}, i}(y ; M) L_{\tau_{b}, j}(\tau ; N) \triangleq \Psi_{y_{b}, M}(y)^{T} \boldsymbol{\Theta} \Psi_{\tau_{b}, N}(\tau),
$$

in which $\boldsymbol{\Theta}=\left[\theta_{(i-1)(j-1)}\right]$ is a matrix with $(M+1) \times(N+1)$ entries as

$$
\begin{aligned}
\theta_{(i-1)(j-1)} & =\sum_{r=0}^{M} \sum_{l=0}^{N} \theta\left(\frac{y_{b}}{M} r, \frac{\tau_{b}}{N} l\right) L_{y_{b}, i}\left(\frac{y_{b}}{M} r ; M\right) L_{\tau_{b}, j}\left(\frac{\tau_{b}}{N} l ; N\right), \\
1 & \leq i \leq M+1,1 \leq j \leq N+1 .
\end{aligned}
$$

\section{Matrix relationships}

Here and in what follows, we give some matrix relationships related to the orthonormal shifted DLPs.

Theorem 4.1 ([44]) Differentiation of the vector $\Psi_{\tau_{b}, N}(\tau)$ introduced in (3.6) satisfies the relation

$$
\frac{d \Psi_{\tau_{b}, N}(\tau)}{d \tau}=\mathbf{D}_{N}^{\left(1, \tau_{b}\right)} \Psi_{\tau_{b}, N}(\tau),
$$


where $\mathbf{D}_{N}^{\left(1, \tau_{b}\right)}=\left[d_{i j}^{\left(1, \tau_{b}\right)}\right]$ is a matrix of order $(N+1)$ with entries

$$
d_{i j}^{\left(1, \tau_{b}\right)}= \begin{cases}\frac{1}{\sqrt{\sigma(i-1, N)}} \sum_{l=0}^{N} \sum_{k=1}^{i-1} \sum_{r=1}^{k}(-1)^{k}\left(\begin{array}{c}
i-1 \\
k
\end{array}\right)\left(\begin{array}{c}
i+k-1 \\
k
\end{array}\right) & 2 \leq i \leq N+1, \\
\times \frac{N}{\tau_{b}} \frac{S_{k}^{(r)}}{N^{(k)}} r l^{r-1} L_{\tau_{b}, j-1}\left(\frac{\tau_{b}}{N} l ; N\right), & 1 \leq j \leq i-1, \\
0, & \text { otherwise. }\end{cases}
$$

Moreover, for any integer $n$, we have

$$
\frac{d^{n} \Psi_{\tau_{b}, N}(\tau)}{d \tau^{n}}=\underbrace{\mathbf{D}_{N}^{\left(1, \tau_{b}\right)} \times \mathbf{D}_{N}^{\left(1, \tau_{b}\right)} \times \cdots \times \mathbf{D}_{N}^{\left(1, \tau_{b}\right)}}_{n \text { times }} \Psi_{\tau_{b}, N}(\tau) \triangleq \mathbf{D}_{N}^{\left(n, \tau_{b}\right)} \Psi_{\tau_{b}, N}(\tau) .
$$

Theorem 4.2 Suppose that $\zeta:\left[0, \tau_{b}\right] \longrightarrow(0,1)$ is a given continuous function and $\Psi_{\tau_{b}, N}(\tau)$ is the vector expressed in (3.7). Then we have

$$
{ }_{0}^{H H} D_{\tau}^{\zeta(\tau)} \Psi_{\tau_{b}, N}(\tau) \simeq \mathbf{Q}_{N}^{\left(\zeta, \tau_{b}\right)} \Psi_{\tau_{b}, N}(\tau)
$$

where $\mathbf{Q}_{N}^{\left(\zeta, \tau_{b}\right)}=\left[q_{i j}^{\left(\zeta, \tau_{b}\right)}\right]$ is a matrix of order $(N+1)$ with entries

$$
q_{i j}^{\left(\zeta, \tau_{b}\right)}= \begin{cases}a_{i j}^{\left(\zeta, \tau_{b}\right)}, & 2 \leq i \leq N+1,1 \leq j \leq N+1 \\ 0, & \text { otherwise, }\end{cases}
$$

in which

$$
\begin{aligned}
a_{i j}^{\left(\zeta, \tau_{b}\right)}= & \frac{1}{\sqrt{\sigma(i-1, N)}} \sum_{l=0}^{N} \sum_{k=1}^{i-1} \sum_{m=1}^{k}(-1)^{k}\left(\begin{array}{c}
i-1 \\
k
\end{array}\right)\left(\begin{array}{c}
i+k-1 \\
k
\end{array}\right) \\
& \times \frac{S_{k}^{(m)}}{N^{(k)}} \frac{m ! l^{m}}{1-\zeta\left(\frac{\tau_{b}}{N} l\right)} \mathbf{E}_{1, m+1}\left(\frac{-\left(\frac{\tau_{b}}{N} l\right) \zeta\left(\frac{\tau_{b}}{N} l\right)}{1-\zeta\left(\frac{\tau_{b}}{N} l\right)}\right) L_{\tau_{b}, j-1}\left(\frac{\tau_{b}}{N} l ; N\right) .
\end{aligned}
$$

Proof Regarding (2.3), we have ${ }_{0}^{H H} D_{\tau}^{\zeta(\tau)} L_{\tau_{b}, 0}(\tau ; N)=0$. So, in the matrix $\mathbf{Q}_{N}^{\left(\zeta, \tau_{b}\right)}$, the first row should be zero. Assume $\hat{i} \geq 1$ and $\zeta:\left[0, \tau_{b}\right] \longrightarrow(0,1)$ be a continuous function. From (2.3) and (3.1), we get

$$
\begin{aligned}
{ }_{0}^{H H} D_{\tau}^{\zeta(\tau)} L_{\tau_{b}, \hat{i}}(\tau ; N)= & \frac{1}{\sqrt{\sigma(\hat{i}, N)}} \sum_{k=0}^{\hat{i}} \sum_{m=0}^{k}(-1)^{k}\left(\begin{array}{c}
\hat{i} \\
k
\end{array}\right)\left(\begin{array}{c}
\hat{i}+k \\
k
\end{array}\right)\left(\frac{N}{\tau_{b}}\right)^{m} \frac{S_{k}^{(m)}}{N^{(k)}}{ }^{H H} D_{\tau}^{\zeta(\tau)} \tau^{m} \\
= & \frac{1}{\sqrt{\sigma(\hat{i}, N)}} \sum_{k=1}^{\hat{i}} \sum_{m=1}^{k}(-1)^{k}\left(\begin{array}{c}
\hat{i} \\
k
\end{array}\right)\left(\begin{array}{c}
\hat{i}+k \\
k
\end{array}\right)\left(\frac{N}{\tau_{b}}\right)^{m} \frac{S_{k}^{(m)}}{N^{(k)}} \frac{m !}{1-\zeta(\tau)} \\
& \times \tau^{m} \mathbf{E}_{1, m+1}\left(-\frac{\tau \zeta(\tau)}{1-\zeta(\tau)}\right) .
\end{aligned}
$$


The above result can be approximated as

$$
{ }_{0}^{H H} D_{\tau}^{\zeta(\tau)} L_{\tau_{b}, \hat{i}}(\tau ; N) \simeq \sum_{\hat{j}=0}^{N} \hat{q}_{\hat{i} j}^{\left(\zeta, \tau_{b}\right)} L_{\tau_{b}, \hat{j}}(\tau ; N)
$$

where, regarding (3.5), we have

$$
\begin{aligned}
\hat{q}_{\hat{i} j}^{\left(\zeta, \tau_{b}\right)}= & \left.\sum_{l=0}^{N}\left(\begin{array}{l}
H H \\
0
\end{array} D_{\tau}^{\zeta(\tau)} L_{\tau_{b}, \hat{i}}(\tau ; N)\right) L_{\tau_{b}, \hat{j}}(\tau ; N)\right|_{\tau=\frac{\tau_{b}}{N} l} \\
= & \frac{1}{\sqrt{\sigma(\hat{i}, N)}} \sum_{l=0}^{N} \sum_{k=1}^{\hat{i}} \sum_{m=1}^{k}(-1)^{k}\left(\begin{array}{c}
\hat{i} \\
k
\end{array}\right)\left(\begin{array}{c}
\hat{i}+k \\
k
\end{array}\right) \frac{S_{k}^{(m)}}{N^{(k)}} \frac{l^{m} m !}{1-\zeta\left(\frac{\tau_{b}}{N} l\right)} \\
& \times \mathbf{E}_{1, m+1}\left(-\frac{\left(\frac{\tau_{b}}{N} l\right) \zeta\left(\frac{\tau_{b}}{N} l\right)}{1-\zeta\left(\frac{\tau_{b}}{N} l\right)}\right) L_{\tau_{b}, \hat{j}}\left(\frac{\tau_{b}}{N} l ; N\right) .
\end{aligned}
$$

Eventually, via the change of indices $\hat{i}=i-1$ and $\hat{j}=j-1$, and considering $q_{i j}^{\left(\zeta, \tau_{b}\right)}$ instead of $\hat{q}_{i-1 j-1}^{\left(\zeta, \tau_{b}\right)}$, we obtain

$$
\begin{aligned}
q_{i j}^{\left(\zeta, \tau_{b}\right)}= & \frac{1}{\sqrt{\sigma(i-1, N)}} \sum_{l=0}^{N} \sum_{k=1}^{i-1} \sum_{m=1}^{k}(-1)^{k}\left(\begin{array}{c}
i-1 \\
k
\end{array}\right)\left(\begin{array}{c}
i+k-1 \\
k
\end{array}\right) \frac{S_{k}^{(m)}}{N^{(k)}} \frac{m ! l^{m}}{1-\zeta\left(\frac{\tau_{b}}{N} l\right)} \\
& \times \mathbf{E}_{1, m+1}\left(\frac{-\left(\frac{\tau_{b}}{N} l\right) \zeta\left(\frac{\tau_{b}}{N} l\right)}{1-\zeta\left(\frac{\tau_{b}}{N} l\right)}\right) L_{\tau_{b}, j-1}\left(\frac{\tau_{b}}{N} l ; N\right)
\end{aligned}
$$

for $2 \leq i \leq N+1$ and $1 \leq j \leq N+1$. Thus, the expressed claim is proved.

For example, whenever $\zeta(\tau)=0.5+0.25 \sin (\tau)$, we obtain

$$
\mathbf{Q}_{5}^{(\zeta, 2)}=\left[\begin{array}{cccccc}
0.0 & 0.0 & 0.0 & 0.0 & 0.0 & 0.0 \\
-1.45297090 & 0.61950634 & 0.34505555 & 0.10604946 & -0.02074431 & -0.01425841 \\
-0.07721547 & -1.86890658 & 1.68369319 & 0.62293619 & 0.00763093 & -0.05902572 \\
-0.63012499 & 0.20031328 & -1.56749609 & 2.41761593 & 0.51704726 & -0.09569810 \\
0.16799373 & -0.53602371 & 0.52366685 & -1.58744491 & 2.84614045 & 0.31438495 \\
-0.10105451 & 0.93727364 & -1.13652072 & 0.69242364 & -1.92755571 & 3.29194139
\end{array}\right] .
$$

\section{Computational method}

In order to use the orthonormal shifted DLPs for problem (1.1) with initial and boundary conditions (1.2), we express the unknown solution as

$$
\theta(y, \tau) \simeq \Psi_{y_{b}, M}(y)^{T} \boldsymbol{\Theta} \Psi_{\tau_{b}, N}(\tau)
$$

where $\boldsymbol{\Theta}$ is an $(M+1) \times(N+1)$ matrix, and its elements are undetermined. Theorem 4.1 results in

$$
\theta_{y y}(y, \tau) \simeq \Psi_{y_{b}, M}(y)^{T}\left(\mathbf{D}_{M}^{\left(2, y_{b}\right)}\right)^{T} \boldsymbol{\Theta} \Psi_{\tau_{b}, N}(\tau)
$$

Besides, Theorem 4.2 together with the above relations yields

$$
{ }_{0}^{H H} \partial_{\tau}^{\zeta(\tau)} \theta(y, \tau) \simeq \Psi_{y_{b}, M}(y)^{T} \boldsymbol{\Theta} \mathbf{Q}_{N}^{\left(\zeta, \tau_{b}\right)} \Psi_{\tau_{b}, N}(\tau)
$$


and

$$
{ }_{0}^{H H} \partial_{\tau}^{\zeta(\tau)} \theta_{y y}(y, \tau) \simeq \Psi_{y_{b}, M}(y)^{T}\left(\mathbf{D}_{M}^{\left(2, y_{b}\right)}\right)^{T} \boldsymbol{\Theta} \mathbf{Q}_{N}^{\left(\zeta, \tau_{b}\right)} \Psi_{\tau_{b}, N}(\tau)
$$

In addition, we represent $\varphi(y, \tau)$ using the orthonormal shifted DLPs as follows:

$$
\varphi(y, \tau) \simeq \Psi_{y_{b}, M}(y)^{T} \Phi \Psi_{\tau_{b}, N}(\tau)
$$

where $\Phi$ is an $(M+1) \times(N+1)$ given matrix, and its elements are evaluated like in (3.8). By inserting (5.2)-(5.5) into (1.1), we obtain

$$
\Psi_{y_{b}, M}(y)^{T}(\underbrace{\boldsymbol{\Theta} \mathbf{Q}_{N}^{\left(\zeta, \tau_{b}\right)}-\mu\left(\mathbf{D}_{M}^{\left(2, y_{b}\right)}\right)^{T} \boldsymbol{\Theta} \mathbf{Q}_{N}^{\left(\zeta, \tau_{b}\right)}-v\left(\mathbf{D}_{M}^{\left(2, y_{b}\right)}\right)^{T} \boldsymbol{\Theta}-\Phi}_{\boldsymbol{\Lambda}}) \Psi_{\tau_{b}, N}(\tau) \simeq 0 .
$$

The functions given in (1.2) can also be approximated via the orthonormal shifted DLPs as

$$
\hat{\theta}(y) \simeq \Psi_{y_{b}, M}(y)^{T} \hat{\boldsymbol{\Theta}}
$$

and

$$
\begin{aligned}
& \tilde{\theta}_{0}(\tau) \simeq \tilde{\boldsymbol{\Theta}}_{0}^{T} \Psi_{\tau_{b}, N}(\tau), \\
& \tilde{\theta}_{1}(\tau) \simeq \tilde{\boldsymbol{\Theta}}_{1}^{T} \Psi_{\tau_{b}, N}(\tau),
\end{aligned}
$$

in which $\hat{\boldsymbol{\Theta}}$ is an $(M+1)$-order column vector, $\tilde{\boldsymbol{\Theta}}_{0}$ and $\tilde{\boldsymbol{\Theta}}_{1}$ are $(N+1)$-order column vectors, and their elements are evaluated like in (3.5). Now, from (1.2), (5.1), (5.7), and (5.8), we obtain

$$
\Psi_{y_{b}, M}(y)^{T}(\underbrace{\boldsymbol{\Theta} \Psi_{\tau_{b}, N}(0)-\hat{\boldsymbol{\Theta}}}_{\Pi_{1}}) \simeq 0
$$

and

$$
(\underbrace{\Psi_{y_{b}, M}(0)^{T} \boldsymbol{\Theta}-\tilde{\boldsymbol{\Theta}}_{0}^{T}}_{\Pi_{2}}) \Psi_{\tau_{b}, N}(\tau) \simeq 0, \quad(\underbrace{\Psi_{y_{b}, M}\left(y_{b}\right)^{T} \boldsymbol{\Theta}-\tilde{\boldsymbol{\Theta}}_{1}^{T}}_{\Pi_{3}}) \Psi_{\tau_{b}, N}(\tau) \simeq 0 .
$$

Utilizing (5.6), (5.9), and (5.10), we generate the following system:

$$
\begin{cases}{[\boldsymbol{\Lambda}]_{i j}=0,} & i=1,2, \ldots, M-1, j=2,3, \ldots, N+1, \\ \left(\Pi_{1}\right)_{i}=0, & i=1,2, \ldots, M+1, \\ \left(\Pi_{2}\right)_{j}=0, \quad\left(\Pi_{3}\right)_{j}=0, & j=2,3, \ldots, N+1 .\end{cases}
$$

Finally, by solving (5.11) and finding the elements of the matrix $\boldsymbol{\Theta}$, we find a numerical solution for the primary VO fractional problem by inserting $\Theta$ into (5.1). 


\section{Numerical examples}

The approach generated using the orthonormal shifted DLPs is applied in this section for solving some numerical examples. The $L_{2}$-error of the numerical results is measured as

$$
e_{\theta}=\left(\int_{0}^{\tau_{b}} \int_{0}^{y_{b}}(\theta(y, \tau)-\tilde{\theta}(y, \tau))^{2} d y d \tau\right)^{1 / 2}
$$

where $\theta$ and $\tilde{\theta}$ are the analytic and numerical solutions, respectively. The convergence order $(\mathrm{CO})$ of this approach is computed as follows:

$$
\mathrm{CO}=\log _{\frac{\bar{N}_{1}}{\bar{N}_{2}}}\left(\frac{\varepsilon_{2}}{\varepsilon_{1}}\right)
$$

where $\varepsilon_{i}$ and $\varepsilon_{2}$ are the first and second $L_{2}$-error values, respectively. Furthermore, $\bar{N}_{i}=$ $\left(M_{i}+1\right) \times\left(N_{i}+1\right)$ for $i=1,2$ is the number of the orthonormal shifted DLPs utilized in the $i$ th implementation. In addition, we have applied Maple 18 (with 15 digits precision) for obtaining the results. Meanwhile, the series generating the Mittag-Leffler function is applied for 25 terms.

Example 1 Consider problem (1.1) on $[0,3] \times[0,1]$ with $\mu=v=1$ and

$$
\varphi(y, \tau)=\sin (\tau) \sinh (y-3)
$$

This example has the analytic solution

$$
\theta(y, \tau)=\sin (\tau) \sinh (3-y)
$$

So, we have

$$
\theta(y, 0)=0, \quad \theta(0, \tau)=\sin (\tau) \sinh (3), \quad \theta(3, \tau)=0 .
$$

We have applied the expressed method for this example with three choices of $\zeta(\tau)$. The extracted results are listed in Table 1. This table shows the high-precision of the proposed approach in solving this example. It also confirms that the results have a high degree of convergence. The last column of this table confirms the low computational works of the pre-

\begin{tabular}{|c|c|c|c|c|c|c|c|c|}
\hline \multirow[t]{2}{*}{ M } & \multirow[t]{2}{*}{$N$} & \multicolumn{2}{|c|}{$\zeta(\tau)=0.50+0.25 \sin (\tau)$} & \multicolumn{2}{|c|}{$\zeta(\tau)=0.85-0.25 e^{-\tau}$} & \multicolumn{2}{|c|}{$\zeta(\tau)=0.65+0.25 \tau^{3} \cos (\tau)$} & \multirow[t]{2}{*}{ CPU time } \\
\hline & & $e_{\theta}$ & $\mathrm{CO}$ & $\overline{e_{\theta}}$ & $\mathrm{CO}$ & $\overline{e_{\theta}}$ & $\mathrm{CO}$ & \\
\hline 5 & 4 & 4.8716E-03 & - & 4.8703E-03 & - & 4.8704E-03 & - & 02.54 \\
\hline 6 & 5 & 4.3022E-04 & 07.2127 & 4.3018E-04 & 07.2122 & 4.3018E-04 & 07.2122 & 05.29 \\
\hline 7 & 6 & $3.2230 \mathrm{E}-05$ & 09.0078 & $3.2171 \mathrm{E}-05$ & 09.0139 & $3.2179 \mathrm{E}-05$ & 09.0130 & 12.71 \\
\hline 8 & 7 & $2.6413 \mathrm{E}-06$ & 09.9541 & $2.6412 \mathrm{E}-06$ & 09.9470 & 2.6379E-06 & 09.9529 & 31.04 \\
\hline 9 & 8 & $1.9235 \mathrm{E}-07$ & 11.7400 & $2.1424 \mathrm{E}-07$ & 11.2568 & $2.1424 \mathrm{E}-07$ & 11.2512 & 64.64 \\
\hline
\end{tabular}
sented algorithm. Graphical behaviors of the extracted results for $\zeta(\tau)=0.50+0.25 \sin (\tau)$ where $(M=9, N=8)$ are illustrated in Fig. 1 . This figure shows the high accuracy of the presented method for obtaining a smooth solution for this example.

Table 1 Results extracted via the presented approach for Example 1 with three choices of $\zeta(\tau)$ 

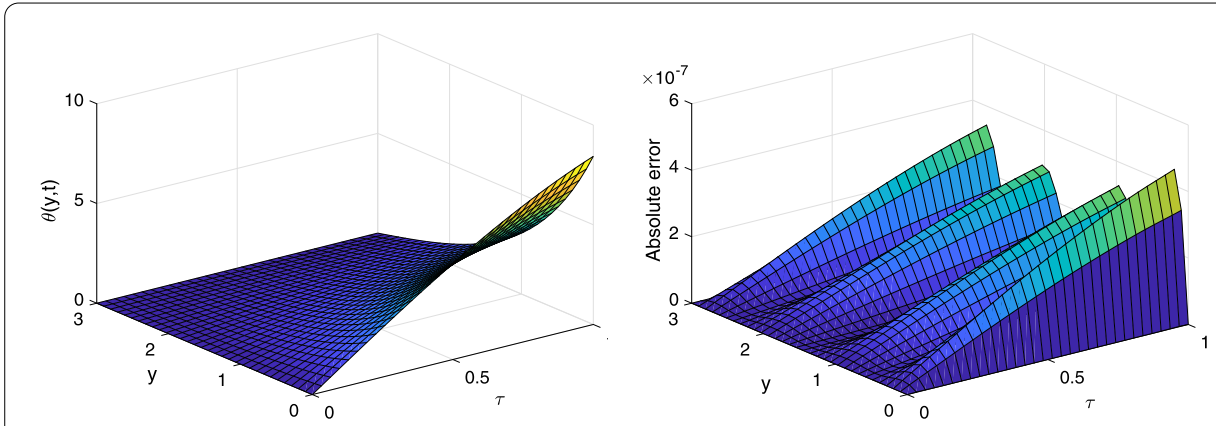

Figure 1 Achieved results for $\theta(y, t)$ whenever $\zeta(\tau)=0.50+0.25 \sin (\tau)$ with $(M=9, N=8)$ in Example 1

Table 2 Results extracted via the presented approach for Example 2 with three choices of $\zeta(\tau)$

\begin{tabular}{|c|c|c|c|c|c|c|c|c|}
\hline \multirow[t]{2}{*}{ M } & \multirow[t]{2}{*}{$N$} & \multicolumn{2}{|c|}{$\zeta(\tau)=0.50+0.25 \sin (\tau)$} & \multicolumn{2}{|c|}{$\zeta(\tau)=0.85-0.25 e^{-\tau}$} & \multicolumn{2}{|c|}{$\zeta(\tau)=0.65+0.25 \tau^{3} \cos (\tau)$} & \multirow[t]{2}{*}{ CPU time } \\
\hline & & $\overline{e_{\theta}}$ & $\mathrm{CO}$ & $e_{\theta}$ & $\mathrm{CO}$ & $\overline{e_{\theta}}$ & $\mathrm{CO}$ & \\
\hline 4 & 4 & 1.6710E-03 & - & $1.6731 \mathrm{E}-03$ & - & $1.6713 \mathrm{E}-03$ & - & 05.70 \\
\hline 5 & 5 & $1.6538 \mathrm{E}-04$ & 06.3430 & $1.6555 \mathrm{E}-04$ & 06.3436 & 1.6540E-04 & 06.3431 & 12.96 \\
\hline 6 & 6 & $6.7121 \mathrm{E}-06$ & 10.3935 & $6.7288 \mathrm{E}-06$ & 10.3887 & $6.7221 \mathrm{E}-06$ & 10.3890 & 23.78 \\
\hline 7 & 7 & 6.8627E-07 & 08.5388 & $6.8598 \mathrm{E}-07$ & 08.5496 & $6.8298 \mathrm{E}-07$ & 08.5623 & 51.53 \\
\hline 8 & 8 & 1.5974E-08 & 15.9628 & $1.5855 \mathrm{E}-08$ & 15.9928 & $1.5414 \mathrm{E}-08$ & 16.0939 & 92.15 \\
\hline
\end{tabular}

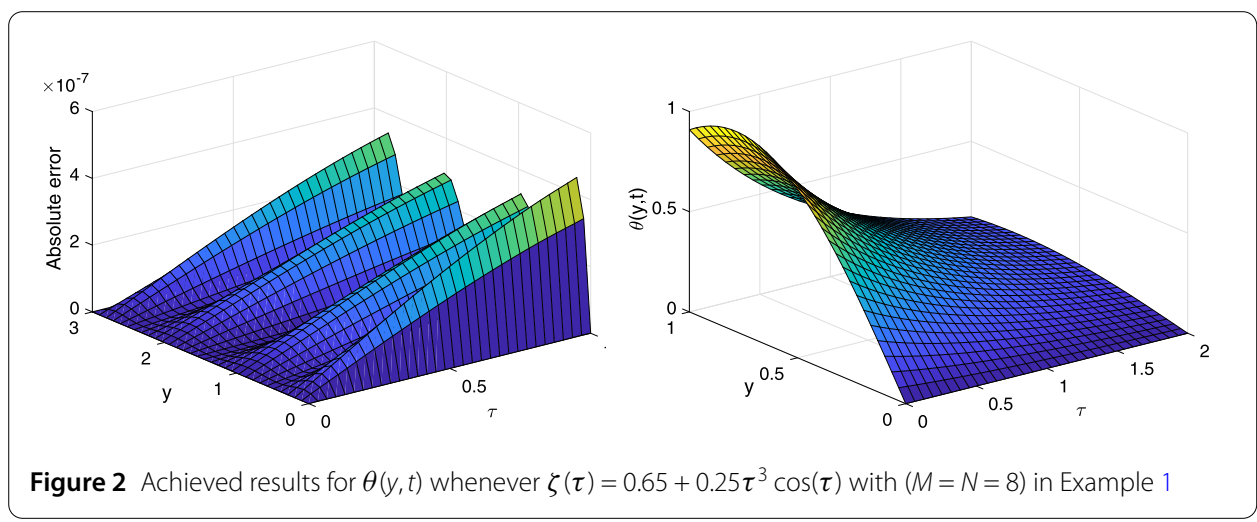

Example 2 Consider problem (1.1) on $[0,1] \times[0,2]$ with $\mu=\frac{1}{2}, v=1$ and

$$
\varphi(y, \tau)=\left(4 e^{-\tau}-\frac{1}{1-\zeta(\tau)} \sum_{l=0}^{\infty}(-\tau)^{l+1} \mathbf{E}_{1, l+2}\left(-\frac{\tau \zeta(\tau)}{1-\zeta(\tau)}\right)\right) \sin (2 y)
$$

This example has the analytic solution

$$
\theta(y, \tau)=e^{-\tau} \sin (2 y)
$$

Thus, we have

$$
\theta(y, 0)=\sin (2 y), \quad \theta(0, \tau)=0, \quad \theta(1, \tau)=e^{-\tau} \sin (2) .
$$

The technique established upon the orthonormal shifted DLPs is implemented for this example. The gained results are provided in Table 2 , and they confirm the high-precision and 
low computations of the approach. It can also be seen that as the number of the orthonormal shifted DLPs increases, the accuracy of the results increases rapidly. The obtained results with $(M=N=8)$ whenever $\zeta(\tau)=0.65+0.25 \tau^{3} \cos (\tau)$ are shown in Fig. 2 . This figure illustrates that the proposed method can provide a highly accurate solution for this example across the domain.

\section{Conclusion}

In this study, the Heydari-Hosseininia fractional differentiation as a kind of nonsingular variable-order (VO) fractional derivative was utilized for generating a VO fractional version of the Sobolev equation. The orthonormal shifted discrete Legendre polynomials (DLPs) as a convenient family of basis functions were employed to generate a numerical algorithm for this equation. A new fractional operational matrix related to VO fractional differentiation of these polynomials was obtained. The established scheme converts solving the problem under consideration into solving an algebraic system of equations. The validity of this technique was investigated by solving two numerical examples. The obtained results confirmed that the established method is able to generate numerical solutions with high accuracy for such problems even by applying a small number of the orthonormal shifted DLPs. As future research direction, the VO fractional derivative applied in this study can be utilized for generating $\mathrm{VO}$ fractional version of other applicable problems, such as Schrödinger equation and advection-diffusion equation.

\section{Acknowledgements}

The authors would like to thank the anonymous reviewers for their valuable comments and suggestions on this paper.

\section{Funding}

The financial assistance is not applicable.

Availability of data and materials

Data sharing is not applicable to this study.

Competing interests

The authors declare that they have no competing interests.

Authors' contributions

All authors contributed equally to the writing of this paper. All authors read and approved the final manuscript.

\section{Author details}

${ }^{1}$ Department of Mathematics, Shiraz University of Technology, Shiraz, Iran. ${ }^{2}$ Faculty of Natural and Agricultural Sciences, University of the Free State, Bloemfontein, South Africa. ${ }^{3}$ Department of Medical Research China Medical University Hospital, China Medical University, Taichung, Taiwan.

\section{Publisher's Note}

Springer Nature remains neutral with regard to jurisdictional claims in published maps and institutional affiliations.

Received: 21 April 2021 Accepted: 21 May 2021 Published online: 26 May 2021

\section{References}

1. Baleanu, D., Ghanbari, B., Asad, J., Jajarmi, A., Mohammadi Pirouz, H.: Planar system-masses in an equilateral triangle: numerical study within fractional calculus. Comput. Model. Eng. Sci. 124(3), 953-968 (2020)

2. Sadat Sajjadi, S., Baleanu, D., Jajarmi, A., Mohammadi Pirouz, H.: A new adaptive synchronization and hyperchaos control of a biological snap oscillator. Chaos Solitons Fractals 138, 109919 (2020)

3. Baleanu, D., Jajarmi, A., Sadat Sajjadi, S., Asad, J.H.: The fractional features of a harmonic oscillator with position-dependent mass. Commun. Theor. Phys. 72(5), 055002 (2020)

4. Podlubny, I.: Fractional Differential Equations: An Introduction to Fractional Derivatives, Fractional Differential Equations, to Methods of Their Solution and Some of Their Applications. Elsevier, Amsterdam (1998)

5. Rouzegar, J., Vazirzadeh, M., Heydari, M.H.: A fractional viscoelastic model for vibrational analysis of thin plate excited by supports movement. Mech. Res. Commun. 110, 103618 (2020)

6. Li, M.: Multi-fractional generalized Cauchy process and its application to teletraffic. Physica A (2020). https://doi.org/10.1016/j.physa.2019.123982 
7. Li, M.: Three classes of fractional oscillators, symmetry-Basel. Symmetry 10(2), 40 (2018)

8. El-Shahed, M., Nieto, J.J., Ahmed, A.: Fractional-order model for biocontrol of the lesser date moth in palm trees and its discretization. Adv. Differ. Equ. 2017, 295 (2017)

9. Veeresha, P., Prakasha, D.G., Singh, J., Kumar, D., Baleanu, D.: Fractional Klein-Gordon-Schrödinger equations with Mittag-Leffler memory. Chin. J. Phys. 68, 65-78 (2020)

10. Veeresha, P., Prakasha, D.G., Singh, J., Kumar, D., Baleanu, D.: Analysis of fractional blood alcohol model with composite fractional derivative. Chaos Solitons Fractals 140, 110127 (2020)

11. Azin, H., Mohammadi, F., Heydari, M.H.: A hybrid method for solving time fractional advection-diffusion equation on unbounded space domain. Adv. Differ. Equ. 2020(1), 1 (2020)

12. Hooshmandasl, M.R., Heydari, M.H., Cattani, C.: Numerical solution of fractional sub-diffusion and time-fractional diffusion-wave equations via fractional-order Legendre functions. Eur. Phys. J. Plus 131(8), 1-22 (2016)

13. Do, Q.H., Ngo, H.T.B., Razzaghi, M.: A generalized fractional-order Chebyshev wavelet method for two-dimensional distributed-order fractional differential equations. Commun. Nonlinear Sci. Numer. Simul. 95, 105597 (2021)

14. Singh, J., Kumar, D., Purohit, S.D., Mishra, A.M., Bohra, M.: An efficient numerical approach for fractional multidimensional diffusion equations with exponential memory. Numer. Methods Partial Differ. Equ. 37(2), $1631-1651(2021)$

15. Srivastava, H.M., Dubey, V.P., Kumare, R., Singh, J., Kumar, D., Baleanu, D.: An efficient computational approach for a fractional-order biological population model with carrying capacity. Chaos Solitons Fractals 138, 109880 (2020)

16. Singh, J., Ahmadian, A., Rathore, S., Kumar, D., Baleanu, D., Salimi, M., Salahshour, S.: An efficient computational approach for local fractional Poisson equation in fractal media. Numer. Methods Partial Differ. Equ. 37, 1439-1448 (2021)

17. Liu, J.G., Yang, X.J., Feng, Y.Y., Cui, P.: On group analysis of the time fractional extended ( $2+1)$-dimensional Zakharov-Kuznetsov equation in quantum magneto-plasmas. Math. Comput. Simul. 178, 407-421 (2020)

18. Liu, J.G., Yang, X.J., Feng, Y.Y., Cui, P., Gengab, L.L.: On integrability of the higher dimensional time fractional KdV-type equation. J. Geom. Phys. 160, 104000 (2021)

19. Coimbra, C.F.M.: Mechanics with variable-order differential operators. Ann. Phys. 12(11-12), 692-703 (2003)

20. Kobelev, Y.L., Klimontovich, Y.L.: Statistical physics of dynamic systems with variable memory. Dokl. Phys. 48, 285-289 (2003)

21. Sun, H.G., Chen, W., Chen, Y.Q.: Variable order fractional differential operators in anomalous diffusion modeling. Physica A 21, 4586-45920 (2009)

22. Heydari, M.H., Avazzadeh, Z., Yang, Y., Cattani, C.: A cardinal method to solve coupled nonlinear variable-order time fractional sine-Gordon equations. Comput. Appl. Math. 39(2) (2020)

23. Hosseininia, M., Heydari, M.H., Roohi, R., Avazzadeh, Z.: A computational wavelet method for variable-order fractional model of dual phase lag bioheat equat. J. Comput. Phys. 395, 1-18 (2019)

24. Roohi, R., Hosseininia, M., Heydari, M.H.: A wavelet approach for the variable-order fractional model of ultra-short pulsed laser therapy. Eng. Comput. (2021). https://doi.org/10.1007/s00366-021-01367-x

25. Babaei, A., Jafari, H., Banihashemi, S.: Numerical solution of variable order fractional nonlinear quadratic integro-differential equations based on the sixth-kind Chebyshev collocation method. J. Comput. Appl. Math. 377, 112908 (2020)

26. Heydari, M.H., Avazzadeh, Z:: Orthonormal Bernstein polynomials for solving nonlinear variable-order time fractional fourth-order diffusion-wave equation with nonsingular fractional derivative. Math. Methods Appl. Sci. (2020). https://doi.org/10.1002/mma.6483

27. Hassani, H., Avazzadeh, Z., Tenreiro Machado, J.A.: Solving two-dimensional variable-order fractional optimal control problems with transcendental Bernstein series. J. Comput. Nonlinear Dyn. 14(6), 061001 (2019)

28. Heydari, M.H., Avazzadeh, Z.: New formulation of the orthonormal Bernoulli polynomials for solving the variable-order time fractional coupled Boussinesq-Burger's equations. Eng. Comput. (2020). https://doi.org/10.1007/s00366-020-01007-w

29. Hosseininia, M., Heydari, M.H., Avazzadeh, Z.: Numerical study of the variable-order fractional version of the nonlinear fourth-order 2D diffusion-wave equation via 2D Chebyshev wavelets. Eng. Comput. (2020) https://doi.org/10.1007/s00366-020-00995-z

30. Nikan, O., Avazzadeh, Z.: A localisation technique based on radial basis function partition of unity for solving Sobolev equation arising in fluid dynamics. Appl. Math. Comput. 401, 126063 (2021)

31. Abbaszadeh, M., Dehghan, M.: Interior penalty discontinuous Galerkin technique for solving generalized Sobolev equation. Appl. Numer. Math. 154, 172-186 (2020)

32. Barenblatt, G.I., Zheltov, I.P., Kochina, I.: Basic concepts in the theory of seepage of homogeneous liquids in fissured rocks strata. J. Appl. Math. Mech. 24(5), 1286-1303 (1960)

33. Ting, T.W.: A cooling process according to two-temperature theory of heat conduction. J. Math. Anal. Appl. 45(1), 23-31 (1974)

34. Dehghan, M., Shafieeabyaneh, N., Abbaszadeh, M.: Application of spectral element method for solving Sobolev equations with error estimation. Appl. Numer. Math. 58, 439-462 (2020)

35. Haq, S., Ghafoor, A., Hussain, M., Arifeen, S.: Numerical solutions of two dimensional Sobolev and generalized Benjamin-Bona-Mahony-Burgers equations via Haar wavelets. Comput. Math. Appl. 72(2), 565-575 (2019)

36. Oruç, O.: A computational method based on Hermite wavelets for two-dimensional Sobolev and regularized long wave equations in fluids. Numer. Methods Partial Differ. Equ. 34(5), 1693-1715 (2018)

37. Liu, J., Li, H., Liu, Y.: Crank-Nicolson finite element scheme and modified reduced-order scheme for fractional Sobolev equation. Numer. Funct. Anal. Optim. 39(15), 1635-1655 (2018)

38. Heydari, M.H., Hosseininia, M.: A new variable-order fractional derivative with non-singular Mittag-Leffler kernel: application to variable-order fractional version of the 2D Richard equation. Eng. Comput. (2020). https://doi.org/10.1007/s00366-020-01121-9

39. Moradi, L., Mohammadi, F.: A discrete orthogonal polynomials approach for coupled systems of nonlinear fractional order integro-differential equations. Tbil. Math. J. 12(3), 21-38 (2019)

40. Gong, D., Wang, X., Wu, S., Zhu, X.: Discrete Legendre polynomials-based inequality for stability of time-varying delayed systems. J. Franklin Inst. 356, 9907-9927 (2019) 
41. Salehi, F., Saeedi, H., Moghadam Moghadam, M.: A Hahn computational operational method for variable order fractional mobile-immobile advection-dispersion equation. Math. Sci. 12, 91-101 (2018)

42. Salehi, F., Saeeidi, H., Mohseni Moghadam, M.: Discrete Hahn polynomials for numerical solution of two-dimensional variable-order fractional Rayleigh-Stokes problem. Comput. Appl. Math. 37, 5274-5292 (2018)

43. Heydari, M.H., Avazzadeh, Z.: Numerical study of non-singular variable-order time fractional coupled Burgers' equations by using the Hahn polynomials. Eng. Comput. (2020). https://doi.org/10.1007/s00366-020-01036-5

44. Heydari, M.H., Avazzadeh, Z., Atangana, A.: Orthonormal shifted discrete Legendre polynomials for solving a coupled system of nonlinear variable-order time fractional reaction-advection-diffusion equations. Appl. Numer. Math. 161 425-436 (2021)

45. Heydari, M.H., Avazzadeh, Z., Cattani, C.: Discrete Chebyshev polynomials for nonsingular variable-order fractional KdV Burgers' equation. Appl. Numer. Math. 44(2), 2158-2170 (2021)

46. Heydari, M.H., Razzaghi, M., Avazzadeh, Z:: Orthonormal shifted discrete Chebyshev polynomials: application for a fractal-fractional version of the coupled Schrödinger-Boussinesq system. Chaos Solitons Fractals 143, 110570 (2021)

47. Heydari, M.H., Avazzadeh, Z., Cattani, C.: Numerical solution of variable-order space-time fractional KdV-Burgers-Kuramoto equation by using discrete Legendre polynomials. Eng. Comput. (2020). https://doi.org/10.1007/s00366-020-01181-x

\section{Submit your manuscript to a SpringerOpen ${ }^{\circ}$ journal and benefit from:}

- Convenient online submission

- Rigorous peer review

- Open access: articles freely available online

- High visibility within the field

- Retaining the copyright to your article

Submit your next manuscript at $\boldsymbol{\nabla}$ springeropen.com 